\title{
$\mathrm{DOE} / \mathrm{ER} / 05007--27$ \\ DE91 004189
}

Progress Report

to

Department of Energy

Contract No. DE-AC02-78ER05007

\section{PARTICLE THEORY AND COSMOLOGY}

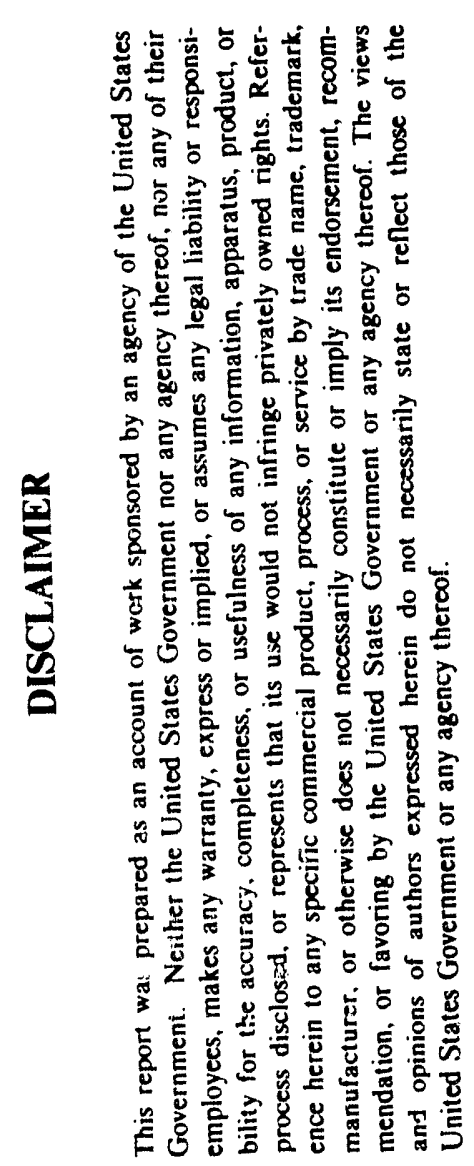

\author{
Submitted by \\ Bartol Research Institute \\ University of Delaware \\ Newark, Delaware 19716 \\ (302) 451-8111
}




\begin{abstract}
The overall objective of the research supported by this contract is to further our understanding of the basic building blocks of matter as well as the role fu.udamental interactions play in cosmolngy and astrophysics. Astrophysical data, such as from high energy cosmic rays and large scale structure of the universe, are employed to constrain particle physics theories. Particle collisions at Tevatron and higher (SSC) energies are also under investigation.

During the past year a systematic reanalysis of the correlation between solar activity and the solar neutrino flux was undertaken. The conclusion seems to be that the Homestake experimental data show a correlation at a significant level, supporting the hypothesis that the neutrino possesses a magnetic moment. A separate, but related, theoretical investigation of electromagnetic properties of elementary particles has led to the discovery of a class of models in which the neucrino is endowed with an appreciable magnetic moment while its remains small. Altogether members of the group have been co-authors of 28 papers during the grant year on topics ranging from fermion masses to the role of ultra-high energy hadronic interactions in cosmic ray physics.
\end{abstract}

Note: In the following sections, references to our own work are numbered to correspond to our "Publications During Current Grant Year" Section F. Other references are assembled at the end of each section.

\title{
A. Non-Accelerator Physics and Particle Phenomenology (T.K. Gaisser)
}

The emphasis of this work is on interpretation of major non-accelerator experiments and their implications for particle physics, cosmic rays, astrophysics and cosmology. Energies of interest extend up to $10^{18} \mathrm{eV}$, equivalent to $\sqrt{s} \sim 40 \mathrm{TeV}$. Extrapolation of minimum bias physics to SSC energies is an integral part of the work. During the past year this work has focussed on interpretation of the underground muons. Gaisser visited Rome during the spring semester of 1990 as guest of the INFN-Rome MACRO group to work on this and related problems.

\section{Underground Muons}

Underground detectors of large area are particularly well-suited for the study of multiple coincident muons. In fact, muons constitute the dominant signal in these detectors. The relative rates of events with different multiplicity reflect the chemical composition of the primary cosmic rays in the 
$10^{14}-10^{16} \mathrm{eV}$ range, which is at present not accessible to direct measurement because of the extremely low intensity of the radiation. Composition as a function of energy in this energy range is of great interest because the spectrum appears to have a feature (the "knee") and because the composition can distinguish among models for the origin and propagation of these high energy particles in the Galaxy. In addition, observation of unexpected phenomena in deep detectors (such as very large muon separations or very high multiplicity bundles) could signal the onset of new processes involving direct lepton production. Another aim of the calculations is thus to provide a definition of 'unexpected' in this context.

Interpretation of measured rates of underground multiple muon events necessarily depends heavily on simulations. Incident cosmic ray nuclei of high energy generate cascades in the atmosphere including charged pions, which sometimes decay to muons before they reinteract. At the same total energy per particle, heavy nuclei with $E>10^{15} \mathrm{eV}$ produce more muons than do primary protons because the relerrant energy per particle ( several $\mathrm{TeV}$ ) is reached more quickly (i.e. higher in the atmosphere) where the pions are more likely to decay. This is the basic fact that must be exploited to obtain information about the composition of the primary cosmic rays. Interpretation is complicated, however, by the many sources of fluctuations that are present. First, there is a spectrum of incident cosmic ray energies, which itself is not measured directly. In addition, the multiplicity of muons is uncertain to the extent that the multiplicity and momentum distributions of pions produced in hadronic interactions are uncertain. Another essential complication arises because any finite detector only intercepts a portion of the shower front, so the relation between the number of muons present in a shower and the number detected depends on the lateral distribution of the muons.

The first problem can be overcome to some extent by measuring the surface shower in coincidence with the underground muons. This is being done at Homestake, Soudan 2 [Das Gupta et al., 1990], and (with the greatest sensitivity) at Gran Sasso by the EASTOP-MACRO collaboration [Bellotti et al., 1990]. In papers presented at the Adelaide Cosmic Ray Conference [5,6], Gaisser, Lipari (U. of Rome) and Stanev conclude that it should be possible to discriminate between two commonly used assumptions about composition with less than one year's running of EASTOP with one MACRO supermodule-and correspondingly more quickly with a more complete MACRO. In a paper presented at Adelaide [7], Auriemma (Rome), Gaisser and Stanev discussed the sensitivity of the underground detector alone to primary composition.

The conclusion one reaches depends, however, on the accuracy of the assumptions that go into the calculation-in particular, on the hadronic interaction model which determines the distributions of multiplicity and of transverse and longitudinal momentum. For this reason we have been working to refine the hadronic interaction model used for the calculations. The 
interaction model used in the original 1985 paper that has been referred to by several of the underground experiments was a simple phenomenological parametrization of accelerator data on hadron-hadron and hadron-nucleus collisions with emphasis on the momentum distribution of the leading nucleon and the inclusive cross section for pion and kaon production, but with no attempt to tune the multiplicity distribution or the correlations among particles beyond what is required by energy conservation.

Gaisser and Stanev have joined with a group from Frascati (Forti, Bilokon, D'Ettorre-Piazzoli, Satta) to use an interaction model developed in Forti's thesis work to parametrize rates of underground multiple muons and their lateral distributions for comparison with the 1985 paper of Gaisser and Stanev. This model is a generalization to hadron-nucleus collisions of the UA5 eveni generator [Alner et al., 1987] for proton-antiproton collisions. The multiplicity distributions are tuned to the data, and the transverse momentum distributions have power law tails. A paper describing this model and its application to multiple muons is to be published in Physical Review [2]. Results are very similar to those obtained in the 1985 paper. Gaisser, Lipari and Stanev are now working on a more ambitious hadronic event generator which will have application to interpretation of cascades at much higher energy as well as to the multiple muon experiments. This will be discussed below in the section on hadron phenomenology.

\section{Atmospheric Neutrinos}

During the previous grant period, in collaboration with Giles Barr (CERN) and Todor Stanev we completed a Monte Carlo calculation of atmospheric neutrino fluxes including effects of muon polarization (Barr et al. 1989). These fluxes are used to establish the background for the proton decay search and to establish the beam for a neutrino oscillation search with atmospheric neutrinos. The Kamioka, IMB and Frejus groups have used the fluxes as input to their detector Monte Carlo codes. The Kamioka group [Hirata et al., 1988] continues to see a somewhat higher $\nu_{e} / \nu_{\mu}$ ratio than they expect from our calculated fluxes including polarization (a $2.2 \sigma$ difference, $L$. 'Totsuka, private communication). Frejus data is consistent with expectation (L. Moscoso, et al., 1989). The IMB analysis appears to be consistent with Kamioka, but a discrepancy with calculation is not claimed.

During the present grant period (since 1 A pril 1990) we have not worked further on calculations of neutrino fluxes, though we have followed developments in the field. We compared our neutrino flux calculations with those of several other groups during the Adelaide Cosmic Ray Conference. Besides our own, there are three other recent calculations of the fluxes of atmospheric neutrinos: Lee (1989); Honda, et al., (1990); Bugaev \& Naumov, (1989) Although there are some differences in absolute fluxes, especially below one $\mathrm{GeV}$, all calculations agree about the ratios of neutrino flavors. Thus the discrepancy between calculation and experiment is due either to a real physical effect or is a consequence of some uncertainty in the Monte 
Carlo of the detector (as distinguished from the calculation of the fluxes). 


\section{Astrophysical Beam Dumps}

Ground-based high energy gamma ray astronomy is currently the subject of many large experiments around the world, including several air Cherenkov and air shower experiments in this country supported by the high energy physics divisions of DOE and NSF. These observations cover the energy range from $\mathrm{TeV}$ and below (air Cherenkov telescopes) to $\sim \mathrm{PeV}$ (air shower arrays) to $\sim \mathrm{EeV}$ (Fly's Eye). As at lower energies, a primary objective of gamma ray astronomy is to look for regions of cosmic ray activity (e.g. point sources) where accelerated cosmic particles collide with gas to produce neutral secondaries that follow linear trajectories through galactic magnetic fields and so point back to their sources. Results from the ground-based experiments have received a great deal of attention in addition because some of them indicate that the signals appear to be hadron-like rather than photonic. This is sometimes called the muon problem because one symptom is that apparent signals in some cases appear to have more muons than would be expected if they are induced by photons. This intriguing situation has been reviewed by Gaisser in a recent article in Science and by D. Fegan in his rapporteur talk at Adelaide. The related subject of high energy neutrino astronomy was review in a paper presented at the Venice conference in February 1990.[10]

\section{Muon Content of Air Showers}

There are two aspects to this problem: first, the degree to which background proton showers can fluctuate toward low muon content and thus mimic photon showers, and second, the degree to which photon showers may have higher muon content than conventional expectation if the cross section for photons to produce hadrons increases with energy, as suggested by Drees and Halzen (1989) and Gandhi et al. (1990). In collaboration with Halzen ot. al. at Wisconsin, Stanev and Gaisser have simulated a large number of protun and photon showers. Experiments at HERA should be able to test the extent to which the photoproduction cross section increases with energy. In the ineantime, however, we conclude [1] that, even with a generous estimate of the increase of photoproduction, only a small fraction of photon showers would have muon content as large as in showers initiated by hadrons. On the other hand, we have concluded [3] that, if the photoproduction cross section increases only logarithmically with energy, proton showers can be discriminated from photon showers by their muon content to a level approaching 1 in 50,000. In this case, muon content will remain a good, practical discriminator for ground-based gamma astronomy. The latter calculations on muon content of proton showers will be extended and used for analysis of size spectra of $\mathrm{GeV}$ muons, which is relevant to resolution of the primary spectrum anıbiguity mentioned above. 


\section{Hadron Phenomenology}

Work at Bartol on hadron phenomenology is motivated both by the desire to understand the physics of minimum bias hadronic interactions and to be able to extrapolata these interactions to $\sqrt{s}=40 \mathrm{TeV}$ for interpretation of atmospheric cascades at $10^{18} \mathrm{eV}$ and above. (This energy happens to be the typical equivalent center of mass energy for cosmic ray cascades detected by the Fily's Eye experiment in Utah as well as that of SSC.) In the energy range around $10^{15} \mathrm{eV}(\sqrt{s} \sim 1 \mathrm{TeV})$ properises of proton-proton collisions are extremely well-studied in the central region (non-forward angles in the CM system). Multiplicity distributions, jet structure, particle content, etc. are ail well measured. The fragmentaticn region, which is crucial for cascade development, is not accessible in most collider experiments, however. In addition, for interpretation of giant air sh'swer experiments, such as Fly's Eye, it is necessary to extrapolate any hadironic event generator to $\sqrt{s} \sim 50 \mathrm{TeV}$. Finally, cosmic ray cascades in the atmosphere involve collisions with nuclear targets and projectiles.

Apart from its potential astrophysical applications, the exercise of building such an event generator raises questions of considerable interest for particle physics. One example is the energy-dependence of the inelasticity (fraction of energy per interaction available for production of pions, kaons, baryon-antibaryon pairs, etc.). Another is the special properties of nucleusnucleus collisions which are being studied by an expanding program of high energy, heavy ion beams. The goal is a hadronic event generator with a comparable level of detail and power to ISAJET [Paige \& Protopopescu, 1986] and PYTHIA [Bengtsson \& Sjöstrand, 1987] (and FRITJOF for nuclei, ) which emphasizes the fragmentation region, which deals in a consistent way with nuclear targets and projectiles and which efficiently generates events at different energies and with different projectiles as they occur in a high energy cascade. Our approach (Gaisser and Stanev, 1989) to this problem is based on an eikonalized minijet model of hadronic interactions (Durand $\& \mathrm{Pi}, 1989$, Block et al., 1990). It combines the dual parton model (Capella, et al., 1987) for soft hadron-hadron and hadron-nucleus collision with the mini-jet model for semi-hard collisions. Existing programs, such as the Lund Monte Carlo which inspired much of the present algorithm, are not suitable for cascade calculations, in large part because they are designed for generation of a large number of events at the same energy with the same beam and projectile.

A simplified version of the model is already being used to generate events for comparizon with Fly's Eye observarions. One goal is to use Fly's Eye measurements of distribution of depths of maximum of air showers to distinguish among various models in the literature of the energy-dependence of inelasticity in hadronic interactions. The mini-jet model, which accounts both for total cross section and for particle production, predicts a gentle increase of inelasticity with energy [8]. A simpler parton model of hadronic 
collisions in which the incident nucleon cascades through the partons of ine target nucleon (Kopeliovich et al., 1989) leads to a more rapid increase of inelasticity. In contrast, thermodynamic or statistical models of particle production (Friedlander, et al., 1983) predict the opposite, i.e., the inelasticity would decrease. The difference is big and has important implications for background soft and semi-hard particles in SCC events. Inelisticity in $p-p$ collisions is $0.7-0.8$ in one case and only $0.1-0.2$ in the other, a difference of $>20 \mathrm{TeV}$ at $\sqrt{s}=40 \mathrm{TeV}$ ! We hope to be able to discriminate Detween these extremes with existing Fly's Eye data. Preliminary accounts of this work are included in two papers presented at Adelaide, the one in collaboration with the Fly's Eye group [9] and the other with Stanev, Tilav and Voyvodic [8]. Work on this subject will continue and is the subject of Tilav's PhD thesis.

\section{References (Section A)}

C. Albajar et al., (UAI collaboration) Nucl. Phys., B309, 405 (1988).

G.J. Ainer et al., Nucl. Phys., 291, 445 (1987).

G. Auriemma, M. Felcini, P. Lipari and J.L. Stone, Phys. Rev., D37, 665 (1.988).

G. Barr et al., Phys. Rev. D39, 3532 (1989).

R. Bellotti et al., Phys. Rev., D42, 1396 (1990).

H. Bengtsson \& T. Sjöstrand, Comp. Phys. Comm., 46, 43 (1987).

Ch. Berger et al., Phys. Rev., D40, 2163 (1989).

M. Block et al., Phys. Rev., D41, 978 (1990) and references therein.

E.V. Bugaev \& V.A. Naumov, Phys. Letters, B232, 391 (1990).

A. Capella et al., Z. Phys., C33, 541 (1987).

U. Das Gupta et al., Proc. 21st ICRC (Adelaide), 9, 327 (1990).

M. Drees \& F. Halzen, Phys. Rev. Letters, $\underline{61}, 275$ (1988).

L. Durand and H. Pi, Phys. Rev., D40, 1436 (1989) and references therein.

Frejus Collaboration (Ch. Lerger et al.) Phys. Letters, B245, 305 (1990).

E.M. Friedlander et al., Phys. Rev., D28, 2903 (1983). 
A.S. Fruchter, D.R. Stinehring \& J.H. Taylor, Nature, $\underline{333}, 237$ (1988).

T.F. Gaisser and T. Stanev, Phys. Lett. 219B, 375 (1989).

R. Gandhi et al., Phys. Rev., D42, 263 (1990).

K.S. Hirata et al., Phys. Lett., B20, 416 (1988).

M. Honda et al., ICRR-Report-215-90-8, July 4, 1990.

B.Z. Kopeliovich, N.N. Nikolaev, and I.K. Potachnikova, Phys. Rev., D39, 769 (1989).

H. Lee, Chungnam University Preprint CNUPNY-1989-TI (1989).

MACRO Collaboration, paper HE4.5-2, submitted to 21st ICRC, Adelaide (1990).

F.E. Paige \& S.D. Protopopescu, Proc. Snowmass Summer Workshop, p. $320(1986)$. 


\section{B. Particle Physics and Cosmology (Q. Shafi)}

The major thrust of the work is in the area of unified theories (including grand unification and higher dimensional superstring models) and in cosmology. The problems being investigated include development of theoretical frameworks for understanding fermion masses and mixings and the study of superstring models derived from superstring theories. Comparisons between grand unified theories and superstring motivated models are also being elucidated. This research effort involves active collaboration with $\mathrm{G}$. Lazarides of the University of Thessaloniki, Greece.

The research overlaps with, complements and extends the research areas under investigation at Bartol by T.K. Gaisser (High Energy Physics, Cosmic Rays), S.M. Barr (High Energy Physics), D. Seckel (Particle Physics/ Cosmology Interface) and E. Rusjan (Superstring Theories). Useful interactions with A. Halprin, M. Barnhill and C.N. Leung of the Theory Group of the University of Delaware are also anticipated. Three graduate students, XingMin Wang, B. Ananthanarayan and M. Freire are also actively involved in research activities in the general area of unified gauge theories and their applications.

\section{Progress Report}

The 10-dimensional $E_{8} \times E_{8}$ heterotic string theory when compactified on certain Calabi-Yau (C-Y) manifolds yields an effective four dimensional theory with $E_{6}$ unification (when the spin connection is identified with the gauge connection) and three families of chiral fermions. One such C-Y manifold is obtained through the intersection of three algebraic equations in $C P^{3} \times C P^{3}$ divided by a freely acting $Z_{3}$ symmetry.

We have studied several phenomenological models arising from the compactification of the theory on this space, initially restricting ourselves to models possessing no more than a 9 element discrete symmetry. Some of these discrete symmetries are of a special kind called $\mathrm{R}$ symmetries, and they place powerful constraints on the allowed couplings in the stperpotential. This helps in obtaining intermediate scales comparable to the compactification scale. Of all the models we have so far discussed, we consider two to be most significant. They both possess the discrete symmetry $Z_{2} \times Z_{3}$, where $Z_{2}$ is used to define a matter parity for the model. The models we have constructed possess the following breaking chain:

$$
\begin{aligned}
S U(3)^{3} \rightarrow S U(3)_{c} & \times S U(2)_{L} \times S U(2)_{R} \times U(1)_{B-L} \\
& \rightarrow S U(3)_{c} \times S U(2)_{L} \times U(1)_{Y} .
\end{aligned}
$$

The first breaking occurs when certain fields labelled $N$ and $\bar{N}$ acquire non-zero vacuum expectarion values (vevs), and the second when certain fields ramed $\nu^{c}$ and $\bar{\nu}^{c}$ ar quire non-zero vev. 
In ref. (13), both these scales are comparable to the compactification scale. To our knowledge, it is the first time that it has been possible to achieve such high breaking scales in the three generation model. The $\mathrm{R}$ symmetry is subsequently broken spontaneously when certain directions in field space acquire non-zero vevs at a scale $\sim 10^{12}-10^{14} \mathrm{GeV}$. The model is found to pass the tests imposed by proton decay considerations from dimension five operators and the requirements arising from renormalization group analysis vis a vis perturbative unification and $\sin ^{2} \theta_{w}$.

In ref. (14) an attempt is made to incorporate a heavy top quark within the framework of grand unified models. An upper bound on the top quark mass is obtained which, for $m_{s}(1 \mathrm{GeV})=175 \mathrm{MeV}$, yields $m_{t}^{\text {phtys }} \simeq 115 \pm 20$ $\mathrm{GeV}$. Decreasing $m_{s}(1 \mathrm{GeV})$ somewhat leads to significantly larger values ior the top mass.

Ref. (23) discusses how the usual cosmological bound on axions can be significantly relaxed through entropy production. We discuss a scenario in which a large amount of entropy is generaied by decaying particles of mass $1-10 T e V$. We find that nucleosynthesis constrains the decay lifetimes to be less than about 0.03 seconds. Using this limiting lifetime, we derive cosmological limits on the axion mass and find that $m_{a}>9 \times 10^{-8} \mathrm{eV}$ if the axion density is dominated by radiation from strings. This limit is far from the limit $m_{a}<10^{-3}$ based on supernova $1987 \mathrm{~A}$, thus showing clearly a case where axionic strings do not need to be inflated away. If the strings were inflated away, we can relax the axion mass bound to $m_{a}>5 \times 10^{-9} \mathrm{eV}$.

In ref. (17) we explore the consequences of the asymptotic relation $\left|V_{c b}\right| \simeq\left(m_{c} / m_{t}\right)^{1 / 2}$ which can be realized in a class of grand unified (e.g., $S O(10)$ ) models. We employed one loop renormalization group equations for the evolution of the gauge and Yukawa couplings as well as the mixing angles. With a single Higgs doublet in the electroweak region and assuming that $0.040 \leq\left|V_{c b}\right| \leq 0.055$, the top quark is predicted to approximately lie between $180 \mathrm{GeV}$ and $220 \mathrm{GeV}$.

\section{Current Research}

Soon after the appearance of the ten dimensional heterotic $E_{8} \times E_{8}$ superstring theory in 1985 , Witten and collaborators proposed a mathematical framework for compactifying six of the ten spacetime dimensions. The six spacelike dimensions are expected to 'curl' up on a scale which is characterized by the Planck length $\left(10^{-33} \mathrm{~cm}\right)$. These dimensions therefore would not be directly accessible to any foreseeable experiment(s) which probe scales on the order of $10^{-16}-10^{-18} \mathrm{~cm}$. But as we note below, the compactification process yields a number of far reaching cor sequences.

The most prominent example of this 'internal' six dimensional space are the so-called Calabi-Yau (C-Y) manifolds. Witten and co-workers showed that the topological aspects of C-Y spaces are expected to play an essential role in determining many of the key physical implications of superstring the- 
ories. For instance, a topological invariant called the Euler characteristic of the C-Y manifold directly determines the number of quark-lepton families observed in nature! (Recall that the standard $S U(3) \times S U(2) \times U(1)$ model has no explanation whatsoever for the observed family replication.) Similarly, other key physical quantities such as interparticle couplings, particle masses, etc., are related to a variety of topological characteristics of the underlying six dimensional space. The latter may not be directly 'visible', but its effects are expected to be felt in many important areas in the 'low energy' world! Clearly, it is important to construct and systematically analyze suitable C-Y manifolds and study their physical implications. One of the prime goals would be to reproduce, as closely as possible, the observed 'low energy' world and come tip with some predictions that can be experimentally tested.

We have initiated a research program in superstring applications at Delaware. The main outside collaborator has been Prof. George Lazarides (formerly at Rockefeller University and now at the University of Thessaloniki, Greece). Current DOE postdoctoral fellow (E. Rusjan) and two of my graduate students (Xing-Min Wang and B. Ananthanarayan) are also involved in this program.

As a first step in this program Lazarides and I have published a series of papers (attached CV) in which we have analyzed some mailiematical aspects and physical implications of a six dimensional manifold discovered in 1985 by the mathematician Yau. The single most important feature here is that this is the only manifold so far known which gives rise to three quark-leptor families (the number found in nature!). We have identified the symmetries of this manifold (isometries) and shown how, depending on the underlying properties of the six dimensional space, physically distinct four dimensional theories will emerge.

We have identified some key symmetries which should play a particularly important role in phenomenological analysis. For instance, a challenge faced by any supersymmetric (SUSY) theory is to prevent the proton from decaying at essentially the same rate as ordinary weak processes. This disaster is usually prevented by invoking some 'magic' symmetries which make the proton live longer than $10^{32} \mathrm{yr}$, as required by current experiments. Remarkably enough, we have shown that several three generation superstrong models give rise to precisely the desired symmetires. These results have greatly encouraged us to systematically pursue the physical consequences of this class of superstring theories.

\section{References (Section B)}

CDF Collaboration, Fermilab (1990).

Kamiokande Collaboration (1990).

G. Lazarides and Q. Shafi, Bartol preprint BA-89-67 (1989). 
Superstring Theory, Vols. 1 and 2, by M. Green, J. Schwarz and E.

Witten, published by Cambridge University Press. 


\section{Theories with Higher Symmetry (S.M. Larr)}

\section{Theories of CP Violation and their Experimental Consequences.}

In the last year or so there has been an upsurge in interest in the electric dipole moments (edm) of elementary particles partly stimulated $t_{y} \approx$ paper of S. Weinberg (1989). Weinberg pointed out that the neutron edm $\left(d_{n}\right)$ is a sensitive prob of $C P$ violation in the Higgs sector. He argued what a certain dimension 6 gluonic operator gives the dominant effect to $c_{n}$ and that other low dimension operators that are. $\mathrm{P}$ and $\mathrm{T}$ odd are suppressed by several powers of light quark masses. lin a recent paper with $\mathrm{A}$. Zee (ref. 19) it was shown that there are two-loop contributions to some of tivioe operators (cpecifically the quark edm and chromo-edm operato's) that are unsuppressed by higher powers of quark masses and which therefore give contributions competitive with and even larger than Weinbeig's operator. Moreover, and perhaps more significantly, we showed that analogous two loop graphs give sirprisingly large contributions to the electron edm and that $d_{e}$ is an even more sensitive probe of Higgs sector CP violation than $d_{n}$, especially in view of recent dramatic improvements in the bounds on $d_{e}$ (S.K. Lamoreaux et al (1987), D. Cho et al. (1989), and S.A. Murthy et al. (1989), Commins et al. (1990) and anticipated further improvements. Ref. 19 has generated even more interest in the subject of the electron and neutron edms as evidenced for example by the papers of R.G. Leigh et al. (1990), D. Chang et al. (1990), and J. Gunion and D. Wyler (1990), which refer to this paper in their abstracts.

Another aspect of $\mathrm{CP}$ violation of great importance is the strong $\mathrm{CP}$ problem. A considerable amount of my past and recent research has been focussed on alternatives to the Peccei-Quinn mechanism which are based on the idea that CP is a spontaneously broken symmetry. (See Barr and Freire, 1990 and references therein.) This work has been extensively discussed in the reviews of J.E. Kim (1987), H.-Y. Cheng (1988), and R. Peccei (1989), and in my talk at the Blois Conference on CP violation. I was invited to give a review of the strong $\mathrm{CP}$ problem in two lectures at the workshop on CP violation at Brookhaven National Lab in June 1990 (ref. 27). Last year my student E.M. Freire and I studied the question of $\epsilon^{\prime} / \epsilon$ in a class of these non-Peccei-Quinn models.

\section{The Interface of Particle Physics with Cosmology and Astrophysics.}

There has been a great deal of excitement over the last few years over $B$ and $L$ violation in the standard model coming from anomaly effects at high temperature or high energy. (See the work of Kuzmin, Rubakov, and Shaposhnikov (1985), Shaposhnikov (1987, 1988) and Arnold and McLerran $(1987,1988)$ for example.) At temperatures in the early universe near 
or above the weak scale it is now generally thought that such 'sphaleron' processes which violate $B$ and $L$ (but conserve $B-L$ ) would have been in equilibrium and wiped out any asymmetry produced above the weak scale (unless that asymmetry were a B-L asymmetry). This has led to much activity in the area of low-temperature baryogenesis in general. In a recent paper I with R. Sekhar Chivukula and E. Farhi (ref. 18) locked at some interesting possibilities if other conserved (up to anomalies) fermion numbers than $B$ and $\mathrm{L}$ are present in the thecry. We considered primarily the case of technibaryon number which is often conserved in technicolor models, however our considerations were more general. 'Sphaleron' processes instead of setting a linear combination of $B$ and $L$ to zero, then set a linear combination of $B, L$ and technibaryon number to zero. The effect is to establish a technibaryon asymmetry that is comparable to (but generally smaller than because oi a Boltzmann suppression) the baryon asymmetry. Since technibaryons are expected to weigh of order a few $\mathrm{TeV}$ this could make technibaryons (or generally whatever carries the new conserved quantum number) a plausible dark matter candiate.

In a related paper A. Nelson and I (ref. 21) showed that under rather general assumptions a measurement of the mass of the lightest neutrino would place an upper bound on the temperature at which baryogenesis occurred. Specifically $T_{0} \lesssim 10^{12} \mathrm{GeV}\left(1 \mathrm{eV} / \mathrm{m}_{\nu}\right)^{2}$. The point is that the same dimension 5 operator in the effective low-energy theory that leads to majorana neutrino masses leads to scattering processes that violate lepton number. If these are in equilibrium at the same time as 'sphaleron' processes both $B$ and $L$ would be wiped out in general.

An area of great activity in the last few years where particle physics and astrophysics meet is the purported anticorrelation of solar neutrinos with sunspot activity. (See Ref. 25, J. Bieber, D. Seckel, T. Stanev, and G. Steigman (1990).) If this holds up it presents a significant challenge to particle physics. Such an effect can be explained if the electron neutrino has a sizable $\left(\gtrsim 10^{-11} \mu_{B}\right)$ magnetic dipole moment (See M. Voloshin, M. Vysotskii; and L. Okun (1986).) But, generally speaking, modals which produce cuch large neutrino moments would also give the neutrino a mass that is too large by many orders of magnitude. In ref. 22, written with my graduate student E.M. Freire and A. Zee, we propuse an extremely simple mechanism that solves this problem, and we also point out that an old model of $A$. Zee (1980) that is a trivial extension of the standard model actually already incorporates this mechanism. This is rather surprising since several groups had tried to use some version of this old model for this purpose and had thought it necessary to complicate the model in various ways to achieve the desired result, adding 'horizontal' gauge interactions or global symmetries, and a number of new particles. (See e.g., Fukugita and Yanagida (1987); Pabu and R. Mohapatra (1989,1990); Leurer and Marcus (1990); and Babu and Mathur (1990.) However, the original model is sufficient. This had been overlooked, perhaps, partly because the mechanism proposed by us operates 
at the two-loop level. Lincoln Wolfenstein ras moved to write "I was very impressed by your recent paper on neutrino magnetic moments... it states that [A. Zee's] original model... gives a large value for $d_{\nu} / \mu_{\nu}$ without any changes. This is rather remarkable considering all the extensions of the model contrived just to give this value."

\section{The family problem and the problem of quark and lepton masses.}

Barr and Zee $(1977,1978)$ were among the first attempts to find realistic models which predict light fermion masses. The radiative hierarchy ideas of those papers were greatly developed in Barr $(1980,1981)$, where it was first observed that symmetries and radiative effects at the GUT scale (or higher) can lead to 'calculable' radiative masses of light fermions. Racently, in Barr (1990), a further significant advance on these ideas was made. In a very economiral model based on the symmetries of grand unification it was shown how both the qualitative and quantitative features of the quark and lepton masses can be given simple explanations in largely group theoretical terms. The only new particles postulated were an extra family plus mirror family, and the only discrete symmetry employed was a $Z_{2}$ parity under which these extra fermions and the Higgs fields are odd. Among the successes of the model are (a) one family (e, $u$, and d) only acquires mass from loops, (b) the fact that $m_{b} \simeq m_{\tau}$ at the GUT scale but not $m_{s} \simeq m_{\mu}$ or $m_{d} \simeq m_{s}$ emerges naturally (explaining an old puzzle), (c) the top is predicted to be heavy ( $\lesssim 120 \mathrm{GeV}$ ), and (d) 'Fritzschian' relations between KM angles and mass-ratios arise naturally. This model does not suffer from the usual problems of lack of predictions, or bad predictions, or complicated, artificial and ad hoc structure. This year in a longer paper I gave a more detailed treatment of this model and discussed various technical issues in greater depth and explored various possible lines of future development (ref. 20).

\section{Grand Unified Theories and Theories with Higher Symmetry.}

Partly as a spin-off of the papers mentioned in the previous section (ref. 20 ) and the papers of G. Lazarides and Q. Shafi (ref. 14) on an alternative approach to quark and lepton masses in the framework of grand unification, my student E.M. Freire undertook a careful renormalization group analysis of the relation $m_{b} / m_{\tau}$ (at $\left.M_{G U T}\right) \cong 1$. In a large class of theories where $S U(4)_{c}$ and $S U(2)_{R}$ (both subgroups of $S O(10)$ ) break at the same scale (as they would, for example, if the right-handed neutrino masses were primarily responsible for these breakings) one has two independent handles on this unknown scale, $\sin ^{2} \theta_{w}$ and $m_{b} / m_{\tau}$. If the top quark has a large mass, as it does, the resulting large Yukawa couplings have a significant effect on the renormalization group equations deternining $m_{\iota} / m_{\tau}$. By requiring consistency in the two determinations of the scale of $S U(4)_{c}$ breaking one 
can say something about how large $m_{\text {top }}$ can be. This work was supervised by both $Q$. Shafi and me.

\section{References (Section C)}

S.M. Barr, ?'hys. Rev., 21, 1424 (1980); Phys. Rev., D24, 1895 (1981).

S.M. Barr, Phys. Rev. Lett., 64, 353 (1990).

S.M. Barr and A. Zee, Phys. Rev. D17, 1854 (1978); Phys. Rev. D18, 4213 (1978).

J. Bieber, D. Seckel, T. Stanev, and G. Steigman, Bartol preprint BA-90-36.

M. Fukugita and T. Yanagida, Phys. Rev. Lett. 58, 1807 (1987);

K. Babu and V. Mathur, Phys. Lett. 196B, 128 (1987); K. Babu and R. Mohapatra, Phys. Rev. Lett. 63, 228 (1989); University of Maryland preprint UMD-PP-91-040; M. Leurer and N. Marcus, Phys. Lett. $\underline{\text { 237B }}, 81$ (1990).

J. E. Kim, Phys. Rep. 150, June 1987 (sec. 5.4); H.-Y. Cheng, Phys. Rep. 158, Jan. 1988 (sec. 4); R.D. Peccei in CP Violation, ed. C. Jarlskog (World Scientific Press, 1989).

V. Kuzmin, V. Rubakov, M. Shaposhnikov, Phys. Lett. B155, 36 (1985); M. Shaposhnikov, Nucl. Phys. B287, 757 (1987); ㄹ299, 797 (1988); P. Arnold, and L. McLerran, Phys. Rev. D36, 581 (1987); D37, 1020 (1988).

S.K. Lamoreaux, J. Jacobs, B. Heckel, F. Raab, and E.N. Fortson, Phys. Rev. Lett. 59, 2275 (1987); D. Cho, K. Sangster, and E.A. Hinds, Phys. Rev. Lett., 63, 2559 (1989); S.A. Murthy, D. Krause, Jr., Z.L. Li, and L.R. Hunter, Phys. Rev. Lett. $\underline{63}, 965$ (1989).

G. Lazarides and Q. Shafi, Bartol preprint BA-89-67.

R.G. Leigh, S. Paban, R.-M. Xu, University of Texas preprint (1990), U'TTG-27-90; D. Chang, W.-Y. Keung, and T.C. Yuan, Northwestern University preprint (1990), NUHEP-TH-90-22; J. Gunion and D. Wyler, ITP Santa Barbara preprint, 1990.

M.B. Voloshin and M.I. Vysotskii, Tad. Fiz. 44, 845 (1986); L.B. Okun et al., ibid. 44, 677 (1986); L.B. Okun et al., Zh. Exp. Theor. Fiz. 91, 754 (1986).

S. Weinberg, Phys. Rev. Lett. $\underline{63}, 2333$ (1989).

A. Zee, Phys. Lett. 93B, 389 (1980). 


\section{Research for David Seckel}

\section{Progress Report}

The last year has seen continuation and completion of several projects (ref. 24)in the general areas of cosmology and particle astrophysics.

The first set of projects, with Jon Engel (Bartol Fellow) and Anna Hayes (Los Alamos), concerns the response of water Čerenkov detectors to weak neutral currents through interactions with Oxygen nuclei. Most previous work has concentrated either on interactions with protons or deuterons, or on charged currents. In this work, we consider excitations of ${ }^{16} \mathrm{O}$ whose decay includes the emission of one or more photons. This may happen directly if the Oxygen excitation is beiow the threshold for particle emission, or from the excited state of a daughter nucleus after particle emission. Our calculations include interaction cross-sections based on state of the art shell model wave functions, and a phenomenological approach to the deexcitation process based on photoabsorption studies.

We have applied our calculations to two cases: the detection of $\mu$ and/or $\tau$ neutrinos, and the detection of 'hadronic' axions. In Ref. 24 we examine a small window (Turner, 1988) in the axion nucleon coupling which is not ruled out by arguments involving the rapid cooling of SN87A or other astrophysical objects (Raffelt, 1990). We showed that if axions existed with parameters in this window, then one would have expected to see a signal of $\geq 5$ events at Kamiokande. Although this result nominally eliminates this window, uncertainties in the flux of axions produced by the supernova prevents a definitive conclusion.

We are currently writing a longer paper concerning the neutrino signal and including more details of our axion calculations. We find that $\sim .2$ events may have been present in the burst detected from SN87A (Hirata, et al., 1987). The actual number of interactions with Oxygen is more like 10 events, but the detection efficiency is only $3-4 \%$. Thus, with the improvements in the Kamiokande detector since 1987 (Hirata, et al., 1990), a galactic supernova would be expected to produce $\sim 10$ observable events. A delay (or lack thereof) in the neutral current signal would provide information about $\mu$ and/or $\tau$ neutrino masses. We also calculate charged current cross-sections on Oxygen for electron neutrinos and use the results to place limits on the mixing of different flavor neutrinos emitted by SN87A. All these results depend upon the assumed neutrino energy spectrum, and so the constraints derived must be interpreted in the light of some uncertainty concerning predictions for the flux of $\mu$ and $\tau$ neutrinos from supernovae (e.g., Burrows and Myra, 1990)

Another project concerning axions resulted in paper (23) with R. Schaefer, Q. Shafi, and G. Lazarides (Thessalonika). The paper concerns the degree to which cosmological limits on the axion mass may be relaxed by entrcpy production. If particle decay produces entropy after the quark hadron 
phase transition, then the density of axions is diluted. However, this effect cannot be carried too far without erasing the good predictions of big bang nucleosynthesis (BBN). We evaluated the BBN limits on entropy production using a numerical code developed previously (Reno and Seckel, 1988). The results were applied to scenarios where the axions arise from coherent field oscillations and to those where most of the axion density comes from the decay of axionic strings. The conclusion of the paper is that for a general class of models which results in entropy production, there is no necessity for inflation to erase the network of axionic strings which arises from the Peccei-Quinn phase transition (Davis and Shellard, 1988).

A visit by G. Steigman to Bartol prompted an investigation by J. Bieber, T. Stanev, D. Sockel, and G. Steigman (Ohio State Univ.) (25) into the time variability of the solar neutrino flux, as measured by the Homestake Neutrino experiment (Lande and Davis, 1990). Our conclusions were that a) there is a significant (.996 confidence level) correlation between the sunspot number and the ${ }^{37} \mathrm{Ar}$ production rate in the Homestake experiment, and b) that the correlation is improved by about 1 order of magnitude (.9993 confidence level) by simultaneously considering a seasonal variation predicted by the hypothesis that neutrinos possess a magnetic dipole moment and precess in the toroidal magnetic fields of the sun (Voloshin, Vysotskii and Okun, 1986) Although the experiment has run for almost two full solar cycles, the positive correlation is almost all due to the data from the solar cycle starting in 1980 . We find this fact to be very curious, and have no rational explanation for it.

Work is nearing completion on a paper with K. Griest (Center for Particle Astrophysics, Berkeley) on some refinements to the calculation of relic abundances. It is a standard problem in cosmology to calculate how many particles of some species will remain after the Universe expands to the point that the species becomes non-relativistic. In this work we examine three cases where the usual calculations are inadequate due to some accidental degeneracy in particle masses. We find that for each case, if the degeneracy holds at the $\sim 10 \%$ level then previous calculations may be in error by more than an order of magnitude. Although this does not drastically alter our view of the early Universe, it does indicate some scenarios where calculations must be done more carefully.

T.K. Gaisser, T. Stanev, and I have continued our work on the possibilities for detecting cosmic ray cascades in the solar atmosphere (28). Neutral particles produced in the sun's atmosphere may escape the magnetic environment surrounding the sun and be detected at Earth. The flux of gamma-rays from $\pi^{0}$ decay in the cascades should be observable by the EGRET instrument (Kanbach, et al., 1989) on the GRO sattelite to be launched in November 1990; nowever, the flux of neutrinos is estimated to be slightly less than could be detected by currently planned detectors(e.g., Adams, el al., 1990) We have now completed our calculations of the neutron flux. This effort required the development a new Monte Carlo code to handle the fragmentation of helium into protons and neutrons. Due to the 
difficulty of identifying neutron events in a harsh cosmic ray environment (Earth orbit) the flux we predict is below the current experimental limits for a flux of neutrons from the sun. Despite this null result, it is anticipated that the techniques used will have application to other environments where magnetic fields confine protons in which case neutrons play a crucial role in enhancing the transport of cosmic rays.

\section{References (Section D)}

A. Adams, et al., Proposal to Construct the First Stage of the GRANDE Facility, GRANDE 90-005 (1990)

A. Burrows and E. Myra, Arizona preprint \#90-08 (1990)

R.L. Davis and E.P.S. Shellard, Nucl. Phys., B324, 167 (1989)

K. Hirata, et al., ICR Report 213-90-6 (1990)

K. Hirata et al., Phys. Rev. Lett., 58, 1490 (1987)

H. Hodges and J. Primack, U. C. Santa Cruz preprint, SCIPP 90/12 (1990)

G. Kanbach, et al., Proceedings of the 'Gamma Ray Observatory Science Workshop', ed. W.N. Johnson, pg. 2-1 (1989)

L.A. Kofman and D.Yu. Pogosyan, Phys. Lett., 214B, 508 (1988)

K. Lande and R. Davis, Jr., Highlight talks at the 21st International Cosmic Ray Conference, Adelaide (1990)

G. Raffelt, Physics Reports, to be published (1990)

M.H. Reno and D. Seckel, Phys. Rev. D37, 3441 (1988)

M.S. Turner, Phys. Rev. Lett., $\underline{60}, 1797$ (1988)

M.B. Voloshin, M.I. Vysotskii, and L.B. Okun, Sov. Jour. Nucl. Phys. $\underline{44}, 440(1986)$ 


\section{E. Recent Research (E. Rusjan and X. Wang)}

During the last year or so, we continued the study of superstring compactifications and their predictions for low energy physics. In particular, we were interested in Gepner models [1], i.e., 4-dimensional string theories obtained from the 10-dimensional heterotic string by replacing 6 space dimensions with superconformal theories constructed as tensor products of minimal models. These theories are exact classical 4-dimensional string vacua and they provide remarkable predictive power. For example, the complete spectrum of fields, including the gauge singlets, is calculable, and so are the Yukawa couplings.

In collaboration with Brian Greene and Ronen Plesser we specifically studied the $1.16_{E}^{3}$ Gepner model [2], which corresponds to the most symmetric complex structure of the Schimmrigk manifold [3]. We computed the spectra for several choices of Wilson loops and corresponding gauge groups [4]. One of these examples has alread; been studied in the Calabi-Yau language [5], but only in the case of freely acting dividing symmetry. We find that the case of nonfreely acting dividing symmetry, which is not amenable to the Calabi-Yau technique, leads to a spectrum, which is closer to the standard model. Other examples, in which the shadow $E_{8}^{\prime}$ is also broken, lead to even more attractive specira with no colored junk. The breaking of $E{ }_{8}^{\prime}$ may be desirable also for other reasons, such as supersymmetry breaking [6]. We find that Wilson lines, which break the hidden $E_{8}^{\prime}$ and are associated with nonfreely acting symmetries, generally lead to spectra containing fractionally charged leptons (so-called cryptons).

\section{References (Section E)}

D. Gepner, Phys. Lett. B199 (1987) 380; Nucl. Phys. B296 (1988) 757.

D. Gepner, String Theory on Calabi-Yau Manifolds: The Three Generation Case, Princeton preprint (Dec. 1987), (unpublished).

R. Schimmrigk, Phys. Lett. B193 (1987) 175.

B.R. Greene, R. Plesser, E. Rusjan and X. Wang, Gauge Symmetry Breaking in Supeconformal Orbifolds, (to be submitted).

C. Panagiotakopoulos, Is the Heterotic Superconformal Three-Generation Model Phenomenologically Viable?, CERN preprint, CERN-TH.5546/89. 


\section{F. Publications During Current Grant Year}

1. The Photoproduction Threshold, T.K. Gaisser, F. Hä zzen, T. Stanev and E. Zas, Phys. Lett. B 243, 444 (1990).

2. Simulation of Atmospheric Cascades and Deep Underground Muons, C. Forti, H. Bilokon, 3. d'Ettorre-Piazzoli, T.K. Gaisser, L. Satta and T. Stanev, to be published in Phys. Rev. D.

3. Gamma Astronomy Above $50 \mathrm{TeV}$ with Muon Poor Showers, T.K. Gaisser, T. Stanev, F. Halzen, W.F. Long and E. Zas, to be pubished in Phys. Rev. D.

4. Cosmic Ray Resw'ts on Total Cross Section in Elastic and Diffractive Scattering, (ed. M.M. Block and A.R. White, Proc. Supplement, Nucl. Phys. B) p. 172 (1989).

5. Surface-Underground Coincidences of Large Deep Underground Detectors, T.K. Gaisser, P. Lipari and T. Stanev, Proc. 21st ICRC (Adelaide) vol. 9, p. 323 (1990).

6. Acceptance of An Air Shower Array, T.K. Gaisser, Proc. 21st ICRC (Adelaide) vol. 10, 312 (1990).

7. Multiple Muons in the Gran Sasso Laboratory, G. Auriemma, T.K. Gaisser and T. Stanev, Proc. 21st ICRC (Adelaide) vol. 9, p. 362 (1990).

8. Energy-dependence of Inelasticity in Nucleon-Nucleon and NucleusNucleus Interactions, T.K. Gaisser, T. Stanev, S. Tilav and L. Voyvodic, Proc. 21st ICRC (Adelaide) vol. 8, p. 55 (1990).

9. Measurement of Cosmic Ray Air Shower Development at Energies Above $10^{17} \mathrm{eV}$, G.L. Cassiday et al. (includes T.K. Gaisser and T. Stanev), Nucl. Phys. B. (Proc. Suppl.) 14A, 368 (1990).

10. Prospects for High Energy Neutrino Astronomy, T.K. Gaisser, Second International Workshop on Neutrino Telescopes, (ed. M. Baldo-Coelin, INFN) p. 397 (1990).

11. High Energy Astrophysics, T.K. Gaisser, to appear in Proc. DPF 90 (Rice University).

12. Matter Parities from the First Realization of the Three Generation Tian-Yau Manifold, Q. Shafi, G. Lazarides, Nucl. Phys. B338, 442 (1990). 
13. Three Generation Superstring Models with Discrete R-Symmetries, Q. Shafi, B. Ananthanarayan and G. Lazarides, Nucl. Phys. B339, 67 (1990).

14. Heavy Top Quark in SO(10), Q. Shafi, G. Lazarides, Bartol preprint BA-89-67, ICTP Freprint 90/153 (1990) to appear in Nucl. Phys. B.

15. Particle Physics ard Cosmology Beyond the Standard Model(s): Grand Unification and Three Generation Superstring Models, Q. Shafi, Dark Matter in the Universe, (eds. P. Galeotti and D.N. Schramm), 79 (1990).

16. Primordial Magnetic ! lonopoles in GUTS and Three Generation Superstring Models, Q. Shafi, Dark Matter in the Universe, (ec's. P. Galeotti and D.N. Schramm) 85 (1990).

17. Is $\left|V_{c b}\right| \sim\left(m_{c c} / m_{t}\right)^{1 / 2}$ ? Q. Shafi, E.M. Freire and G. Lazarides, to appear in Mod. Phys. Lett. (1990).

18. Electroweak Fermion Number Violation and the Production of Stable Particles in the Early Universe, S.M. Barr, R. S. Chivukula and E. Farhi, Phys. Lett. 241B, 387 (1990).

19. Electric Dipole Moment of the Electron and of the Neutron, S.M. Barr and A. Zee, Phys. Rev. Lett. 65, 21 (1990).

20. A Predictive Hierarchical Model of Quark and Lepton Masses, S.M. Barr, BA-90-40 (to be published in Phys. Rev. D).

21. Upper Bound on Baryogenesis Scale from Neutrino Mass, A.E. Nelson and S.M. Barr, Phys. Lett. 246B, 141 (1990).

22. A Mechanism for Large Neutrino Magnetic Moments, S.M. Barr, E.M. Freire, A. Zee, Phys. Rev. Lett.65, 2626 (1990).

23. Dilution of Cosmological Axions by Entropy Production, G. Lazarides, R. Schaefer, D. Seckel and Q. Shafi, Nucl. Phys. B, 346, 193 (1990).

24. Emission ald Detectability of Hadronic Axions from SN87A, J. Engel, A. Hayes and D. Seckel, Phys. Rev. Lett. 65,960 (1990).

25. Sunspots and Solar Neutrinos, J.W. Bieber, D. Seckel, T. Stanev and G. Steigman, to appear in Nature (1990).

26. Neutrino Masses, Hadronic Axions, and Supernovae, APS Division of Particles and Fields, D. Seckel, DPF90, Rice University (1990). 
27. The Strong CP Problem, S.M. Barr, 2 lectures in "Proc. of Brookhaven Nat'l Lab Summer Workshop in CP Violation," 1990 (World Scientific, 1991).

28. Signatures of Cosmic Ray Literactions on the Solar Surface, D. Seckel, T. Stanev and T.K. Gaisser, Proc. 21st Int. Cosmic Ray Conf. (Adelaide) vol. 4, 463 (1990). 

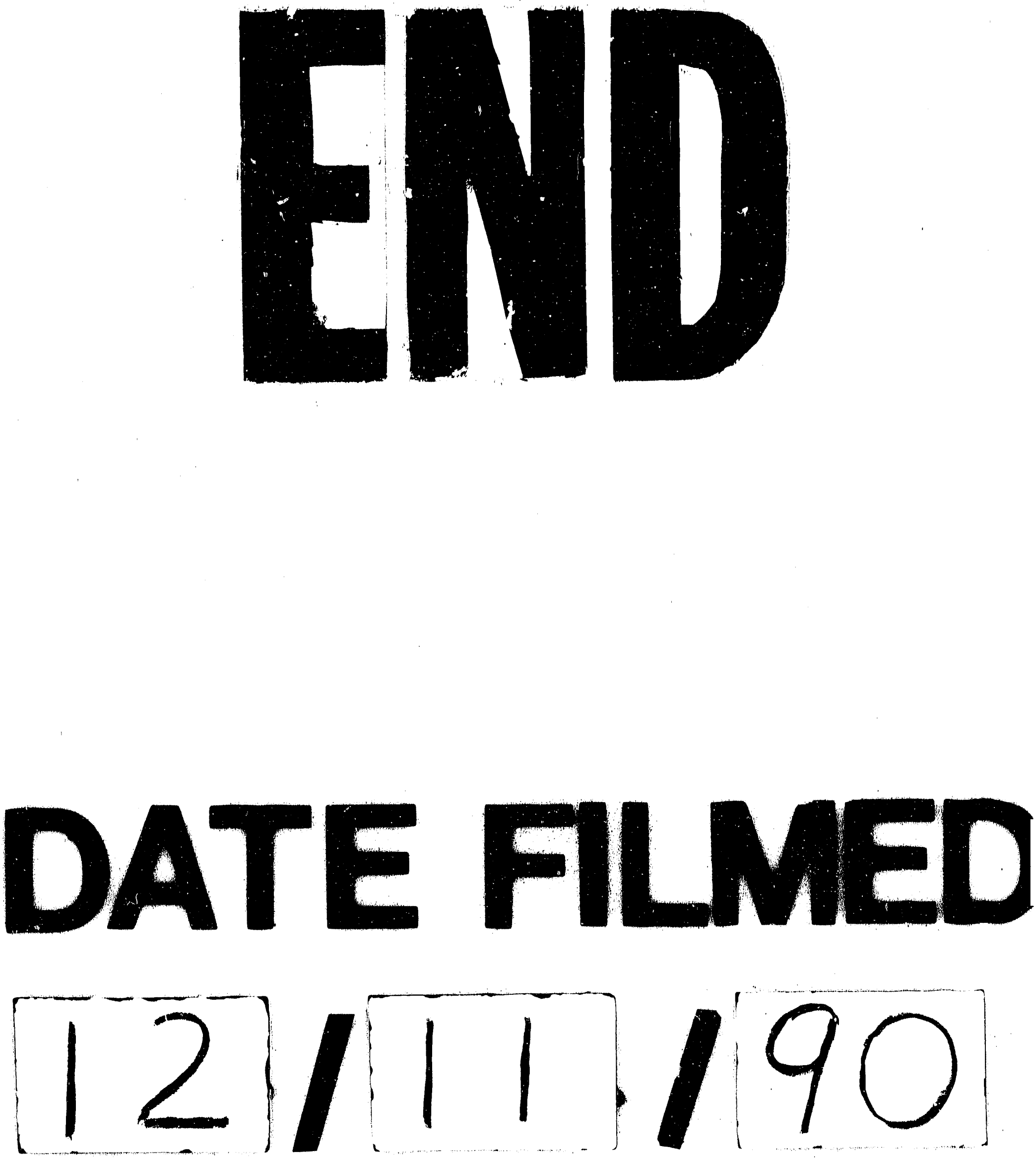


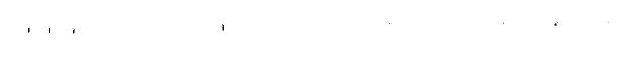

